\title{
Using metagenomic analyses to estimate the consequences of enrichment bias for pathogen detection
}

\author{
James B Pettengill', Eugene McAvoy², James R White ${ }^{3}$, Marc Allard', Eric Brown ${ }^{1}$ and Andrea Ottesen ${ }^{1 *}$
}

\begin{abstract}
Background: Enriching environmental samples to increase the probability of detection has been standard practice throughout the history of microbiology. However, by its very nature, the process of enrichment creates a biased sample that may have unintended consequences for surveillance or resolving a pathogenic outbreak. With the advent of next-generation sequencing and metagenomic approaches, the possibility now exists to quantify enrichment bias at an unprecedented taxonomic breadth.
\end{abstract}

Findings: We investigated differences in taxonomic profiles of three enriched and unenriched tomato phyllosphere samples taken from three different tomato fields $(n=18)$. $16 \mathrm{~S}$ rRNA gene meteganomes were created for each of the 18 samples using 454/Roche's pyrosequencing platform, resulting in a total of 165,259 sequences. Significantly different taxonomic profiles and abundances at a number of taxonomic levels were observed between the two treatments. Although as many as 28 putative Salmonella sequences were detected in enriched samples, there was no significant difference in the abundance of Salmonella between enriched and unenriched treatments.

Conclusions: Our results illustrate that the process of enriching greatly alters the taxonomic profile of an environmental sample beyond that of the target organism. We also found evidence suggesting that enrichment may not increase the probability of detecting a target. In conclusion, our results further emphasize the need to develop metagenomics as a validated culture independent method for pathogen detection.

Keywords: Enrichment bias, Metagenomics, Pathogen, Taxonomy

\section{Findings}

Enrichment procedures are often used to increase the probability of detection of a particular pathogen. However, due to numerous factors including competition, differences in relative growth rates, growth inhibitors, and presence of bacteriophages, [1] enrichment results in a biased sample [i.e., enrichment bias; 2]. Given the objective of enriching, this bias is expected. However, our limited understanding of non-target effects that occur through the enrichment process make it difficult to rely on specific enrichment methods as best diagnostic approaches. Enrichment may have inherent and currently poorly understood consequences on resident microflora of a particular food

\footnotetext{
* Correspondence: andrea.ottesen@fda.hhs.gov

${ }^{1}$ FDA Center for Food Safety and Applied Nutrition, Division of Microbiology, Molecular Methods and Subtyping, 5100 Paint Branch Parkway, College Park, MD 20740, USA

Full list of author information is available at the end of the article
}

or environmental sample, which may prove detrimental to the resolution of public health disease outbreaks.

Historically, quantifying enrichment bias was accomplished by determining the differences in relative abundance of a target organism (e.g., Salmonella spp., Shigella spp., Listeria ssp., or Escherichia spp.) among different enrichment treatments [1,3,4]. With the advent of next-generation sequencing and metagenomic methods, we can now describe the differences in incidence and potential abundance beyond the target organisms to that of nearly all bacterial lineages within a sample; our ability to do so will continue to improve with the increasing sequencing depth provided by next-generation sequencing methods and continually expanding reference databases. Metagenomic approaches also provide insight to ecological and functional dynamics associated with environments that host human pathogens, which in turn may increase our predictive ability to identify where

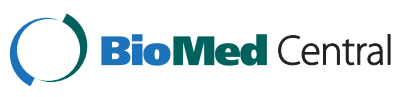


a specific pathogen may arise. Although metagenomics has been used extensively to describe microbial communities, its utility for quantifying enrichment bias for public safety investigative purposes has yet to be fully explored [5].

In this study, we employed next-generation sequencing and a 16S rRNA metagenomic approach to evaluate the ways in which enrichment changes the taxonomic profile of a sample. We also investigate the effects of such a practice on the ability to detect the specific organism targeted by the enrichment procedure (i.e., the first step in the Bacterial Analysis Management (BAM) protocol for detection of Salmonella employed by the United States Food and Drug Administration (USFDA)). Differences in taxonomic profiles were characterized among 18 samples comprising 3 replicates each of enriched through universal preenrichment broth (UPB) and non-enriched tomato phyllosphere samples from three different sites surrounding Immokalee, Florida, USA, which is an area to which outbreak causing strains of Salmonella have been traced. We acknowledge that other culture independent methods exist for pathogen detection [e.g., quantitative PCR; 6,7], however, they are not well suited to quantifying enrichment bias and are not evaluated here.

From the analysis of 165,259 sequences (average number per sample $=9486$; Table 1 ), we found based on a

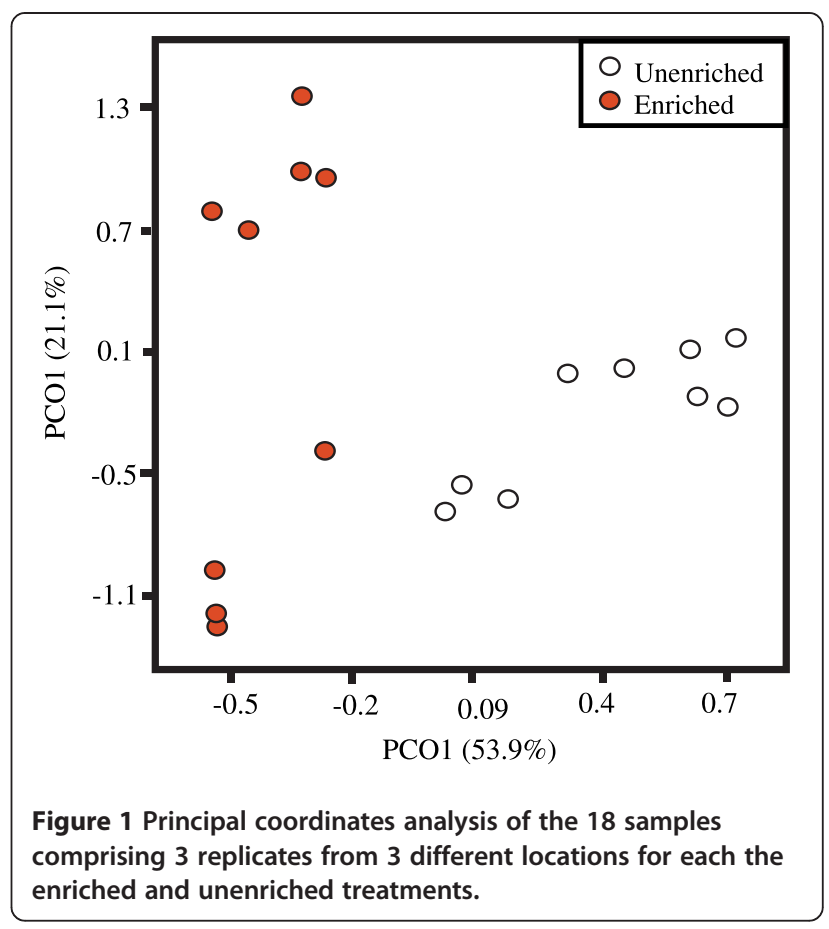

principal coordinates analysis ( $\mathrm{PCoA})$ that enriched and unenriched samples have different taxonomic profiles

Table 1 Sampling locations, the number of cpDNA sequences, the number of sequences, Chao's index, and two estimates of the number of Salmonella sequences for each of the 18 replicates

\begin{tabular}{|c|c|c|c|c|c|c|c|c|c|}
\hline Site & Location & Treatment & Replicate & MG-RAST ID & $\begin{array}{l}\text { cpDNA } \\
\text { Sequences }\end{array}$ & Sequences & Chao's Index & $\begin{array}{l}\text { Salmonella } \\
\text { (Normalized) }\end{array}$ & $\begin{array}{l}\text { Salmonella } \\
\text { (Raw) }\end{array}$ \\
\hline \multirow[t]{6}{*}{ Florida47 } & $2627^{\prime} 42^{\prime \prime} \mathrm{N}$ & Enriched & 1 & 4478869.3 & 0 & 10323 & 182.2 & 0 & 0 \\
\hline & & & 2 & 4478871.3 & 0 & 8576 & 777.1 & 0 & 0 \\
\hline & & & 3 & 4478873.3 & 3 & 9528 & 950.0 & 0 & 0 \\
\hline & $08126^{\prime} 16^{\prime \prime} \mathrm{W}$ & Unenriched & 1 & 4478870.3 & 38 & 5852 & 493.2 & 0 & 0 \\
\hline & & & 2 & 4478872.3 & 74 & 8965 & 414.7 & 0 & 0 \\
\hline & & & 3 & 4478874.3 & 28 & 12235 & 593.7 & 0 & 0 \\
\hline \multirow[t]{6}{*}{ BHN836 } & $2622^{\prime} 05^{\prime \prime} \mathrm{N}$ & Enriched & 1 & 4478875.3 & 0 & 9972 & 1020.5 & 0 & 0 \\
\hline & & & 2 & 4478877.3 & 1 & 9272 & 749.9 & 0 & 0 \\
\hline & & & 3 & 4478879.3 & 1 & 12836 & 660.2 & 0 & 0 \\
\hline & $0811^{\prime} 59^{\prime \prime} \mathrm{W}$ & Unenriched & 1 & 4478876.3 & 78 & 8786 & 848.4 & 0 & 0 \\
\hline & & & 2 & 4478878.3 & 301 & 10804 & 1058.8 & 0 & 0 \\
\hline & & & 3 & 4478880.3 & 195 & 8751 & 816.8 & 0 & 0 \\
\hline \multirow[t]{6}{*}{ Soraya } & $2617^{\prime} 12^{\prime \prime} \mathrm{N}$ & Enriched & 1 & 4478881.3 & 2 & 7850 & 589.6 & 0.50 & 28 \\
\hline & & & 2 & 4478883.3 & 0 & 7901 & 166.1 & 0.10 & 1 \\
\hline & & & 3 & 4478885.3 & 1 & 7358 & 156.0 & 0 & 0 \\
\hline & $08120^{\prime} 21 \mathrm{~W}$ & Unenriched & 1 & 4478882.3 & 1554 & 11911 & 1186.7 & 0 & 0 \\
\hline & & & 2 & 4478884.3 & 2556 & 10382 & 1040.3 & 0 & 0 \\
\hline & & & 3 & 4478886.3 & 669 & 9458 & 915.7 & 0 & 0 \\
\hline Total & & & & & 5501 & 170760 & $701.11^{*}$ & $0.03^{*}$ & $1.61^{*}$ \\
\hline
\end{tabular}

* Averages rather than sums. 
(Figure 1). At the Domain level, there was a statistically significant difference between the treatments in the abundance of Eukaryotes $(P<0.024)$ but not Bacteria $(P=0.367)$. This result is consistent with our results comparing Chao's diversity index between the two treatments, which were not significantly different from one another $(P=0.12$; Table 1$)$.

The treatments differed significantly in the abundance of representatives of three different Bacterial phyla: Firmicutes were greater in the cultured samples $(t=8.28$, $d f=15.90, p<0.001)$; Actinobacteria were greater in the uncultured samples $(t=-8.33, d f=14.66, p<0.001)$; and Proteobacteria were greater in the uncultured samples $(t=-6.57, d f=11.64, p<0.001)$ (Figure 2). The asymmetry in the abundance of taxa within those three phyla and lack of statistically significant differences among the other four bacterial phyla investigated (Bacteroidetes, Spirochaetes, Chloroflexi, and Nitrospirae) likely explains the insignificance of the differences between the treatments at the domain level for Bacteria.

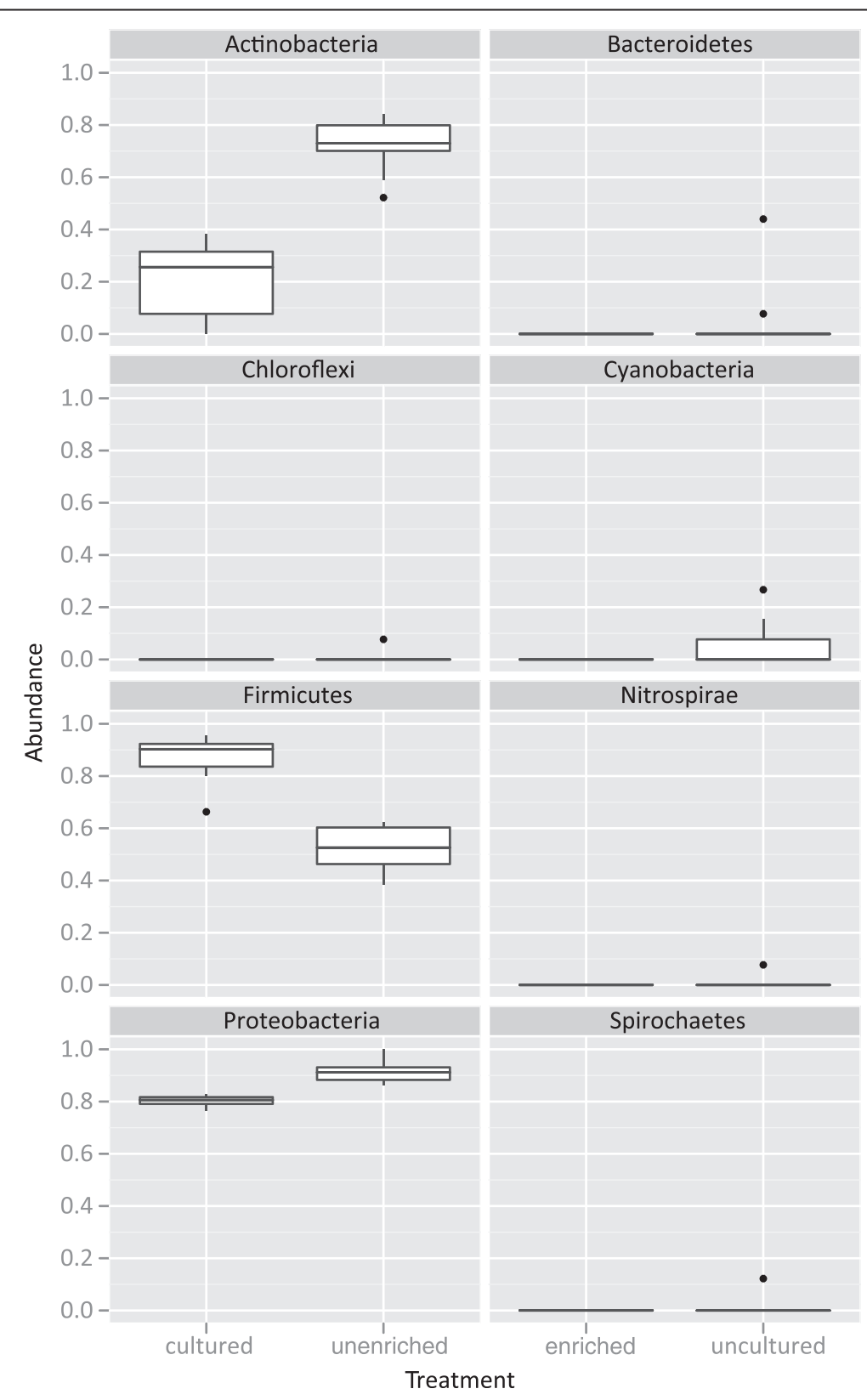

Figure 2 Boxplots illustrating the differences in normalized abundances of sequences assigned at the taxonomic level of bacterial phyla between the enriched and unenriched treatments. 
Focusing on the Enterobacteriaceae, which includes Salmonella, we found evidence for significant differences $(p<0.05)$ in the abundance of five genera between the cultured and uncultured treatments (Enterobacter, Klebsiella, Escherichia, Citrobacter, and Cronobacter).

Out of a total of approximately 54,000 sequences within the enriched samples we only found 28 sequences that were classified as Salmonella based on a best hit classification method. Given that only two of nine enriched samples contained putative Salmonella sequences, there was no significant difference between the two treatments in the abundance of Salmonella ( $p=0.260$; Table 1, Figure 3). We also found through further examination of the taxonomy of those sequences that they were just as likely to represent other species (e.g., Panteoa and/or Klebsiella). No sequences were assigned to Salmonella based on the lowest common ancestor approach. The results from the naïve Bayes classifier also provided evidence that cultured samples contained putative Salmonella sequences. However, we found that based on BLAST results of those

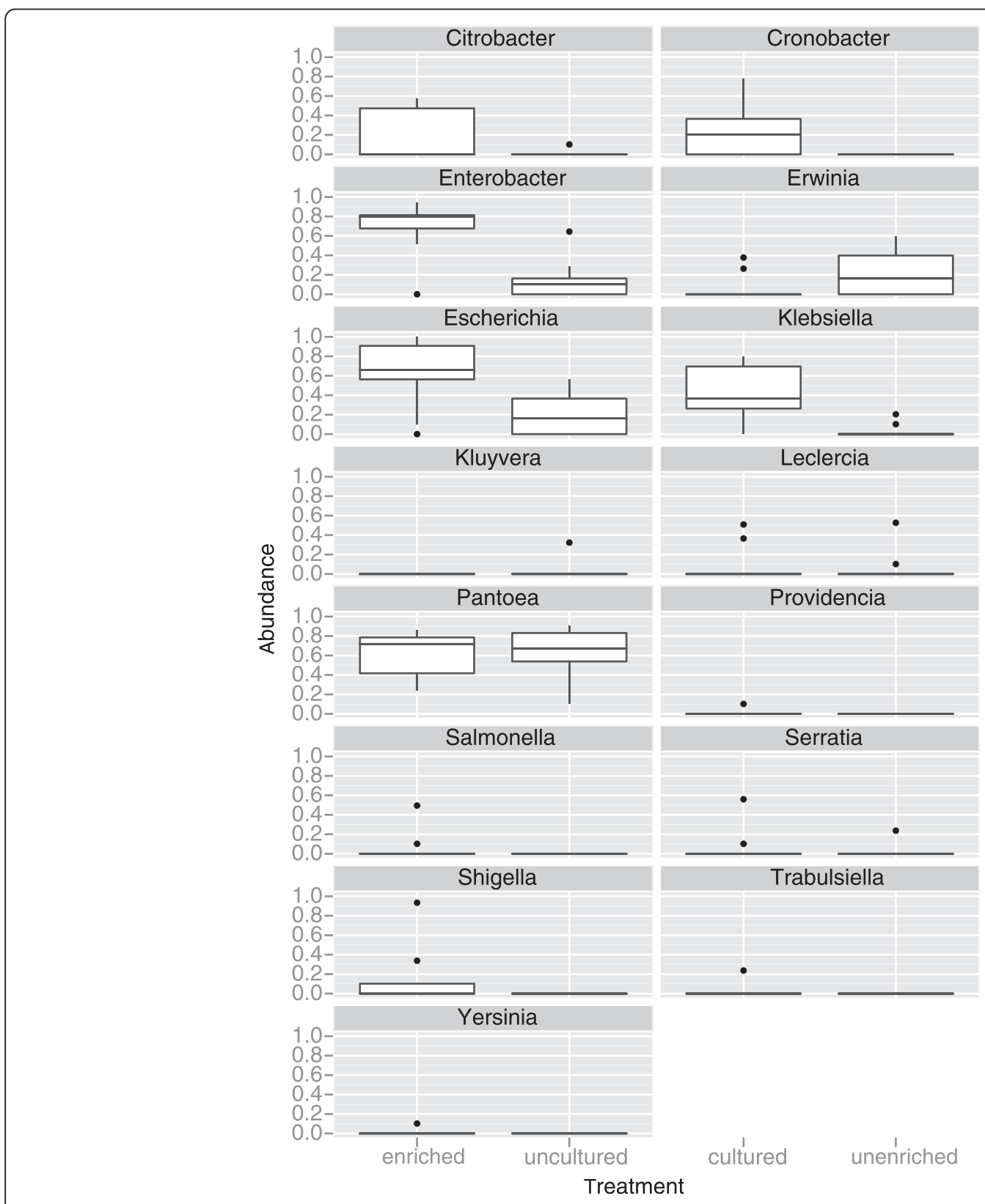

Figure 3 Boxplots illustrating the differences in normalized abundances of sequences assigned at the taxonomic level of genus within the family Bacteriaceae between the enriched and unenriched treatments. 
sequences that they were also assigned to other taxa besides Salmonella and, therefore, we cannot say with certainty that they represent any one taxon. There were no putative Salmonella species found in the uncultured replicates regardless of classification method.

\section{Conclusions}

Our results, which are among the first quantifying enrichment bias using a metagenomic approach, illustrate that the procedure of enriching a sample results in a drastically different taxonomic profile beyond that of the abundance of the target organism. This may not be of concern when there is certainty regarding the cause of an outbreak but if the organism responsible is unknown [so called 'orphan' microbes associated with diseases of previously unknown cause; 8] then our results suggest that enriching could greatly hinder the ability to identify those involved. For example, our results illustrate that enrichment using UPB significantly decreased the number of Actinobacteria, which is a taxonomic group that contains a number of human pathogens (e.g., Tropheryma whipplei). If a member of that group were responsible for an outbreak then our results suggest that the use of UPB may confound our ability to identify the causative agent.

Second, we found that there was no significant difference between cultured and uncultured samples in the putative abundance of the organism, Salmonella, targeted by the first step in the Salmonella enrichment methods recommended by the FDA. The work of Jacobson et al. [9] that found that after following the BAM protocol only two-thirds of 540 samples artificially contaminated with Salmonella were positive for Salmonella further suggests that enrichment may not always result in detecting the target organism. Although it could be argued that with increased sequencing depth we would have detected a significant difference (Figure 4), a greater number of sequence reads could also have resulted in the detection of greater numbers of Salmonella within each treatment. Such a result would further support the results of this study. We expect that future studies will find the latter to be true. Therefore, as sequencing methods are developed that produce an even greater number of reads, metagenomics will likely become a validated tool for pathogen detection decreasing the need for enrichment.

\section{Methods}

\section{Sampling and enrichment}

Tomato samples were collected in May of 2011 from three different locations surrounding Immokalee, Florida, USA (Table 1). All samples were kept separate and brought back to the laboratory for processing. Below we briefly describe the enrichment protocol. For more detailed instructions see http://www.fda.gov/Food/ScienceResearch/LaboratoryMethods/BacteriologicalAnalyticalManualBAM/ucm070149.htm\#Isol. Universal Preenrichment Broth (UPB) was added to samples of tomatoes at approximately 1.0 times the weight of the tomatoes, which was then incubated for $60 \mathrm{~min}$ at room temperature before being incubated at $35^{\circ} \mathrm{C}$ for $24 \mathrm{~h}$.

\section{DNA extraction and PCR amplification}

DNA from uncultured samples was extracted from a wash of tomatoes and leaves. The resulting wash was sonicated for $5 \mathrm{~min}$ before centrifugation to generate a pellet from which DNA was extracted. DNA from cultured samples was extracted from approximately $1 \mathrm{ml}$ of overnight culture that was also spun down to create a pellet. Total DNA was extracted using the

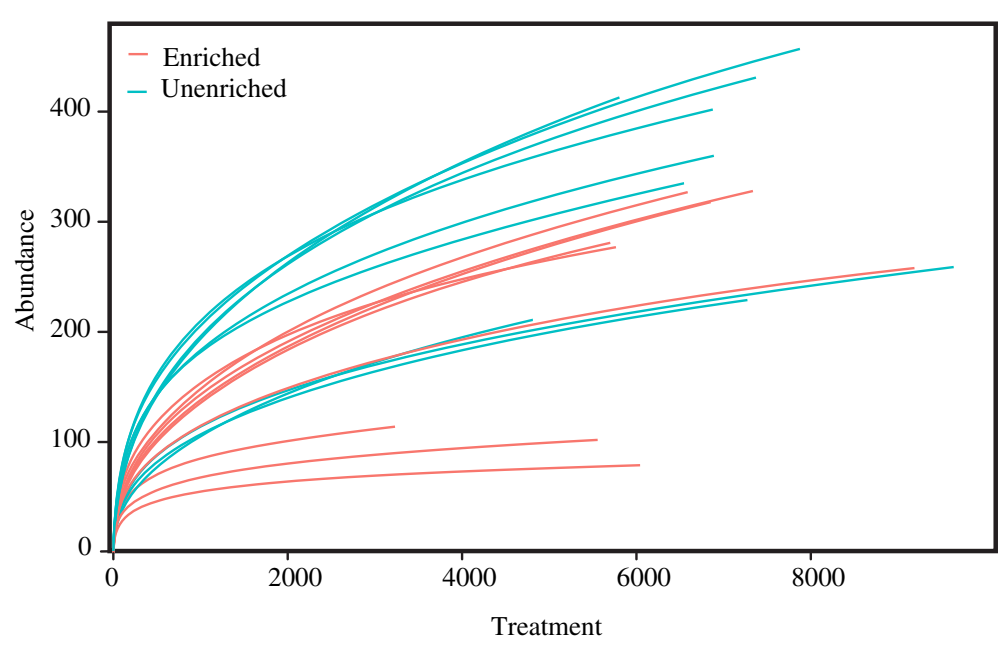

Figure 4 Rarefaction plots depicting the number of species detected as a function of the number sequence reads. 
Promega Wizard DNA Purification Kit according to the manufacturer's specifications.

$16 \mathrm{~S}$ fragments (V1-V3) were amplified for Roche pyrosequencing (454) using Roche Fusion Primer A, key, and MIDs (Multiplex identifiers) 27 through 44, and 27F: 5' CGT ATC GCC TCC CTC GCG CCA TCAG (10 base pair MID) AGA GTT TGA TCC TGG CTC AG $3^{\prime}$ and Roche Fusion Primer B, key, no mid and 533R: 5' CTA TGC GCC TTG CCA GCC CGC TCAG TTA CCG CGG CTG CTG GCA C 3'. Removal of PCR amplicons under 300 bases was performed using AmpPure XP from Agencourt at a ratio of $60 \mu \mathrm{l}$ of AMPure beads to $100 \mu \mathrm{l}$ PCR product. We used the above primers because 1) they created amplicons of suitable length for sequencing on the 454 machine, 2) have been used in previous studies detecting Salmonella [e.g., 10], and 3) have been validated in our lab where they were used to successfully amplify pure cultures of Salmonella ssp. Newport.

It is important to acknowledge that our results are based on analysis of $16 \mathrm{~S}$ ribosomal DNA sequences obtained via PCR dependent methods, which can be considered an enrichment process that introduces its own potential biases. However, we have assumed that whatever bias may have been introduced through targeted sequencing was equal between the treatments and, thus, did not affect our conclusions regarding the effects on taxonomic profiles between the enriched and unenriched treatments. We also acknowledge that extraction procedures may represent another source of bias that can affect the taxonomic profile of a sample [e.g., 11]. Additional studies are necessary to determine whether extraction bias would affect the two treatments, enriched and unenriched, differently.

\section{Emulsion PCR and sequencing}

Amplicons were diluted to $10^{7}$ molecules per $\mu \mathrm{l}$ and pooled to generate a mixture containing an equimolar representation of each independent replicate for subsequent emulsion PCR. Emulsion PCR was done using the Roche Lib-A MV kit according to the manufacturer's specifications.

Approximately 800,000 enriched beads were loaded into one-quarter region of the Roche Titanium FLX pico-titer plate for sequencing on the Titanium FLX platform according to the manufacturer's specifications. Sequencing read numbers were parsed in house with an adapted script to include MIDs beyond the 14 MIDs that Roche software automatically recognizes. Chimeric and chloroplast sequences were removed using the program ClovR [12]. Specifically, 12,913 were detected as being chimeric; 5,501 sequences were identified as being chloroplast DNA by the RDP classifier. Interestingly cultured samples had fewer sequences identified as chloroplast compared to uncultured replicates, however, this result was not significant $(t=-2.0793, d f=8$, $p=0.0712$ ).

\section{Analyses}

Sequences were uploaded into MG-RAST v3.1.2 [13], where they are also publicly available (Table 1). All analyses within MG-RAST were conducted using the following parameter settings: the RDP annotation source, maximum e-value $=1.0^{-5}$, minimum identity cutoff $=98 \%$, minimum alignment length cutoff $=150 \mathrm{bp}$. We constructed rarefaction plots to estimate the limits of detection of our sequencing efforts (i.e., how well we were able to detect the taxonomic diversity within each sample).

As a first step to identify whether the cultured and uncultured replicates had different taxonomic profiles, we conducted a principal coordinates analysis ( $\mathrm{PCoA}$ ) on the normalized abundance counts of taxa within each replicate using the Bray-Curtis dissimilarity index. We also estimated Chao's alpha diversity metric for each replicate using QIIME v1.4.0 [14]. Significance testing of the normalized abundances determined by MG-RAST and Chao's diversity index were conducted grouping samples into two treatments (each with 9 replicates) and using Welch's two-sample $t$-test as implemented in the stats package in R [15]. Using MG-RAST, we also identified the groups at different taxonomic levels that were responsible for the observed differences based on the PCoA. This was accomplished by comparing normalized abundances of a given taxonomic group between the different treatments with significance testing again done using a $t$-test.

Given our emphasis on the probability of detecting Salmonella, we determined the number of samples within each replicate that were identified as such using two different platforms. The first was MG-RAST within which the number of putative Salmonella sequences was determined based on the best-hit classification and lowest common ancestor approaches. The second platform we used was NBC (naïve Bayes classifier) that assigns sequences to species through a Bayesian framework with all bacterial genomes within NCBI serving as the reference database [16]. Because of the limited taxonomic breadth of the database used by NBC, we then used BLASTN [17] and the 'nr' database to further evaluate the taxonomic assignment of putative Salmonella sequences from the NBC analyses.

Competing interests

The authors declare that they have no competing interests.

Authors' contributions

$\mathrm{AO}$ and $\mathrm{EB}$ conceived and designed the experiment. JBP and JRW conducted bioinformatic analyses. EM facilitated sample collection. MA 
provided the use of sequencing equipment. JBP and $\mathrm{AO}$ wrote the manuscript. All authors read and approved the final manuscript.

\section{Acknowledgements}

We are grateful to Cong Li for assistance with the sequencing of the samples. Funding for this research was provided by the U. S. Food and Drug Administration, the Army Research Office and the Oak Ridge Institute for Science and Education fellowship awarded to JBP.

\section{Author details}

'FDA Center for Food Safety and Applied Nutrition, Division of Microbiology, Molecular Methods and Subtyping, 5100 Paint Branch Parkway, College Park, MD 20740, USA. ${ }^{2}$ University of Florida - IFAS Hendry County Extension, PO Box 68, LaBelle, FL 33975, USA. ${ }^{3}$ IGS Institute for Genome Sciences, University of Maryland School of Medicine, 801 West Baltimore St, Baltimore, MD 21201, USA.

Received: 22 March 2012 Accepted: 10 July 2012

Published: 27 July 2012

\section{References}

1. Muniesa M, Blanch AR, Lucena F, Jofre J: Bacteriophages may bias outcome of bacterial enrichment cultures. Appl Environ Microbiol 2005, 71(8):4269-4275

2. Dunbar J, White S, Forney L: Genetic diversity through the looking glass: effect of enrichment bias. Appl Environ Microbiol 1997, 63(4):1326-1331.

3. Singer RS, Mayer AE, Hanson TE, Isaacson RE: Do microbial interactions and cultivation media decrease the accuracy of Salmonella surveillance systems and outbreak investigations? J Food Protect 2009, 72(4):707-713.

4. Davies PR, Turkson PK, Funk JA, Nichols MA, Ladely SR, Fedorka-Cray PJ: Comparison of methods for isolating Salmonella bacteria from faeces of naturally infected pigs. J Appl Microbio/ 2000, 89(1):169-177.

5. Nakamura S, Maeda N, Miron IM, Yoh M, Izutsu K, Kataoka C, Honda T, Yasunaga T, Nakaya T, Kawai J, et al: Metagenomic diagnosis of bacterial infections. Emerg Infect Dis 2008, 14(11):1784-1786.

6. Malorny B, Paccassoni E, Fach P, Bunge C, Martin A, Helmuth R: Diagnostic real-time PCR for detection of Salmonella in food. Appl Environ Microbiol 2004, 70(12):7046-7052.

7. Hadjinicolaou AV, Demetriou VL, Emmanuel MA, Kakoyiannis CK, Kostrikis LG: Molecular beacon-based real-time PCR detection of primary isolates of Salmonella typhimurium and Salmonella enteritidis in environmental and clinical samples. BMC Microbio/ 2009, 9:97.

8. Mortimer PP: Five postulates for resolving outbreaks of infectious disease. J Med Microbiol 2003, 52(Pt 6):447-451.

9. Jacobson AP, Gill VS, Irvin KA, Wang H, Hammack TS: Evaluation of methods to prepare samples of leafy green vegetables for preenrichment with the bacteriological analytical manual Salmonella culture method. J Food Protect 2012, 75(2):400-404.

10. Weisburg WG, Barns SM, Pelletier DA, Lane DJ: 16 S ribosomal DNA amplification for phylogenetic study. J Bacterio/ 1991, 173(2):697-703.

11. Carrigg C, Rice O, Kavanagh S, Collins G, O'Flaherty V: DNA extraction method affects microbial community profiles from soils and sediment. Appl Microbiol Biotechnol 2007, 77(4):955-964.

12. Angiuoli SV, Matalka M, Gussman G, Galens K, Vangala M, Riley DR, Arze C, White JR, White O, Fricke WF: CloVR: a virtual machine for automated and portable sequence analysis from the desktop using cloud computing. BMC Bioinforma 2011, 12:356.

13. Glass EM, Wilkening J, Wilke A, Antonopoulos D, Meyer F: Using the metagenomics RAST server (MG-RAST) for analyzing shotgun metagenomes. Cold Spring Harb Protoc 2010, 2010(1):pdb prot5368.

14. Caporaso JG, Kuczynski J, Stombaugh J, Bittinger K, Bushman FD, Costello EK, Fierer N, Pena AG, Goodrich JK, Gordon Jl, et al: QIIME allows analysis of high-throughput community sequencing data. Nat Method 2010, 7(5):335-336.

15. R Development Core Team: $R$ : a language and environment for statistical computing. R Foundation for Statistical Computing. Vienna, Austria: $R$ Foundation for Statistical Computing; 2008.

16. Rosen $G L$, Reichenberger $E R$, Rosenfeld AM: NBC: the naïve classification tool webserver for taxonomic classification of metagenomic reads. Bioinformatics 2011, 27:127-129.
17. Altschul SF, Gish W, Miller W, Myers EW, Lipman DJ: Basic local alignment search tool. J Mol Biol 1990, 215(3):403-410.

\section{doi:10.1186/1756-0500-5-378}

Cite this article as: Pettengill et al:: Using metagenomic analyses to estimate the consequences of enrichment bias for pathogen detection. BMC Research Notes 2012 5:378.

\section{Submit your next manuscript to BioMed Central and take full advantage of:}

- Convenient online submission

- Thorough peer review

- No space constraints or color figure charges

- Immediate publication on acceptance

- Inclusion in PubMed, CAS, Scopus and Google Scholar

- Research which is freely available for redistribution 